\title{
Cuidado ao paciente com Doença Renal Crônica no nível primário: pensando a integralidade e o matriciamento
}

\author{
Care for patients with Chronic Kidney Disease \\ at the primary healthcare level: considerations \\ about comprehensiveness and establishing a matrix
}

Paulo Félix de Almeida Pena ${ }^{1}$

Aluísio Gomes da Silva Júnior ${ }^{2}$

Paulo de Tarso Ribeiro de Oliveira ${ }^{3}$

Gracyelle Alves Remigio Moreira ${ }^{1}$

Alexandre Braga Libório ${ }^{1}$

${ }^{1}$ Programa de Pósgraduação em Saúde Coletiva, Universidade de Fortaleza (Unifor). Av. Washington Soares 1321/ Bloco S/01, Edson Queiroz. 60811-905 Fortaleza CE. alexandreliborio@yahoo.com.br

${ }^{2}$ Departamento de Planejamento Em Saúde, Instituto de Saúde da Comunidade, Universidade

Federal Fluminense.

${ }^{3}$ Instituto de Filosofia e

Ciências Humanas,

Universidade Federal do

Pará (UFPA).

\begin{abstract}
Chronic kidney disease is a public health problem throughout the world. Early detection and treatment can reduce morbidity, mortality and associated expenditures. The objective of this research is to examine primary healthcare from a physician's perspective, evaluating the patient's access to other levels of healthcare. Questionnaires were given to 62 family physicians working in primary healthcare units in the city of Fortaleza. Glomerular filtration rate was measured by only $8.1 \%$ of the physicians when evaluating patients with diabetes and $4.8 \%$ when evaluating hypertensive patients. The majority of physicians (51.2\%) referpatients with slight/moderate Glomerular filtration rate reduction to a nephrologist. On the other hand, $25.8 \%$ do not refer patients with advanced chronic kidney disease reduction to a specialist. The gap between these levels of healthcare creates a barrier to user access, prejudicing comprehensive healthcare. The creation of new work processes is urgently required and the matrix process is a viable option to connect these healthcare levels for the care of patients with chronic kidney disease or its main risk factors (diabetes and arterial hypertension).
\end{abstract}

Key words Chronic kidney disease, Primary healthcare, Matrix support, Access to healthcare, Comprehensive healthcare
Resumo A Doença Renal Crônica é um problema de saúde pública crescente no mundo. A detecção e o tratamento precoces reduziriam as altas taxas de morbimortalidade e os custos associados. Este trabalho buscou identificar o panorama do acesso ao cuidado a partir da conduta dos médicos da Atenção Primária à Saúde na linha de cuidado da doença. Aplicaram-se questionários para 62 médicos de família dos Centros de Saúde da Família do município de Fortaleza. Os achados apontam que a Taxa de Filtração Glomerular foi mensurada por apenas $8.1 \%$ dos médicos para pacientes diabéti$\cos$ e $4.8 \%$ para pacientes hipertensos. Mais da metade dos médicos (51.2\%) referenciariam o paciente apresentando redução leve/moderada da Taxa de Filtração Glomerular ao nivel secundário. Por outro lado, $25.8 \%$ dos médicos não referenciariam o paciente com Doença Renal Crônica avançada ao especialista. A lacuna entre esses dois níveis da atenção implica em barreira de acesso ao usuário, podendo comprometer avanços no plano da integralidade. A criação de novos dispositivos no processo de trabalho torna-se urgente e o apoio matricial apresenta-se como proposta viável para a articulação das ações entre os níveis da atenção no cuidado do portador da Doença Renal Crônica ou seus fatores de risco.

Palavras-chave Doença renal crônica, Atenção Primária à Saúde, Apoio matricial, Acesso aos cuidados de saúde, Integralidade 


\section{Introdução}

O Sistema Único de Saúde (SUS), com seus princípios e diretrizes, vem sendo construído baseado em um ideário de reformas em que um novo modo de produção de saúde seja capaz de racionalizar a organização e a gestão da atenção à saúde, universalizando seu acesso. O modelo de organização escolhido foi o de Atenção Primária à Saúde (APS), materializado com o nome de Estratégia de Saúde da Família (ESF) ${ }^{1-6}$.

A APS, experiência exitosa de sistemas de saúde em vários países, apresenta-se como organização de redes assistenciais articuladas, onde os serviços de nível primário são os de primeiro contato do usuário. Caracterizam-se pelo acesso facilitado e atuam de forma efetiva sobre os principais problemas de saúde da população. Essa posição enquanto porta de entrada preferencial na rede assistencial permite também desenvolver a coordenação de cuidados pelo médico generalista ou de família ${ }^{7-10}$.

Os médicos de família, além de funcionarem como o profissional de primeiro contato para o maior elenco de situações, controlam as referências para as especialidades, exercendo um papel regulador que deveria ser pautado pela lógica garantidora do acesso ao melhor cuidado possível, conforme um projeto terapêutico definido, considerando as necessidades daquele indivíduo ou comunidade ${ }^{7,9,11}$.

Assim, temos como ponto de partida o acesso a todos os níveis da atenção enquanto instrumento fundamental para a garantia da integralidade, considerando que esse atributo interfere e sofre interferência na organização da equipe multiprofissional, na oferta ampla e na articulação de serviços em níveis crescentes de sofisticação tecnológica, assim como na articulação com outros recursos necessários para a resolução dos problemas de saúde da comunidade ${ }^{4,8,12}$. Entretanto, no Brasil, alguns problemas de considerável gravidade, têm sua assistência em nível primário ainda pouco resolutiva. Destacamos a Doença Renal Crônica (DRC).

A DRC apresenta-se como um problema de saúde pública crescente em todo o mundo, com uma prevalência de $11 \%$ na população adulta norte-americana, associado a altas taxas de morbimortalidade, perda da qualidade de vida e custos para o sistema ${ }^{13-19}$. O conjunto de serviços de saúde deve estar adequado para cuidar desse problema e controla-lo.

A definição da DRC é baseada em três componentes: anatômico ou estrutural; funcional baseado na Taxa de Filtração Glomerular (TFG), marcador que determina o estágio da doença; e temporal (Quadro 1) $)^{18,20-22}$.

Quadro 1. Classificação das Fases da Doença Renal Crônica.

\begin{tabular}{|c|c|c|}
\hline Estágio & Função Renal & TFG (mL/min.) \\
\hline 1 & $\begin{array}{l}\text { - Lesão renal (proteinúria); } \\
\text { - Função preservada; } \\
\text { - Fatores de risco. }\end{array}$ & $>90$ \\
\hline $\begin{array}{l}2 \text { (Insuficiência renal } \\
\text { funcional / leve) }\end{array}$ & $\begin{array}{l}\text { - Creatinina normal; } \\
\text { - Ausência de sintomas clínicos; } \\
\text { - Detecção das anormalidades apenas por exames } \\
\text { acurados (depuração); } \\
\text { - Controle razoável do meio interno. }\end{array}$ & $60-89$ \\
\hline $\begin{array}{l}3 \text { (Insuficiência renal } \\
\text { laboratorial / moderada) }\end{array}$ & $\begin{array}{l}\text { - Paciente ainda clinicamente bem; } \\
\text { - Elevação dos níveis de creatinina e ureia. }\end{array}$ & $30-59$ \\
\hline $\begin{array}{l}4 \text { (Insuficiência renal clínica } \\
\text { / avançada) }\end{array}$ & $\begin{array}{l}\text { - Paciente pode ressentir da disfunção renal; } \\
\text { - Apresenta sinais e sintomas marcados de } \\
\text { uremia. }\end{array}$ & $15-29$ \\
\hline $\begin{array}{l}5 \text { (Fase terminal de } \\
\text { insuficiência renal crônica - } \\
\text { DRCFT) }\end{array}$ & $\begin{array}{l}\text { - Rins perdem controle do meio interno } \\
\text { (incompatibilidade com a vida); } \\
\text { - Paciente intensamente sintomático; } \\
\text { - Métodos de depuração artificial do sangue. }\end{array}$ & $<15$ \\
\hline
\end{tabular}

Fonte: Adaptado Brasil (2006) ${ }^{22}$; Bastos et al. $(2010)^{27}$. 
Os principais grupos de risco para o desenvolvimento da DRC são pacientes portadores de Diabetes Mellitus (DM), Hipertensão Arterial Sistêmica (HAS), idosos e pessoas com história familiar de nefropatia ${ }^{21-23}$.

Estima-se que atualmente $25,5 \%$ das mulheres e $20,7 \%$ dos homens com idade $\geq 18$ anos relataram diagnóstico prévio de hipertensão ${ }^{24,25}$. Já a DM acomete aproximadamente $6 \%$ da população brasileira, configurando-se em um risco de desenvolvimento de nefropatia de cerca de $20 \%{ }^{21}$.

Em relação ao custo, alguns dados do Reino Unido mostram que o número de pacientes que recebem Terapia Renal Substitutiva (TRS) tem aumentado rapidamente custando mais de $2 \%$ do orçamento do Sistema Nacional de Saúde (NHS) ${ }^{26,27}$. O gasto com o programa de diálise e transplante renal no Brasil situa-se ao redor de 1,4 bilhões de reais ao ano ${ }^{21,23}$.

Todo paciente pertencente aos chamados grupos de risco, mesmo que assintomático, deve ser avaliado anualmente com exame de urina, para detectar perda de proteína, e creatinina sérica (Cr); como conduta de triagem para prevenção e diagnóstico precoce de DRC. Preconiza-se também a utilização de equações preditivas para o cálculo da TFG ${ }^{18,20,22,26,28-30}$.

Portanto, a detecção da DRC pode se realizar pelos controles laboratoriais rotineiros e de baixo custo que médicos da APS realizam periodicamente em seus pacientes hipertensos e diabéti$\cos ^{24,28,29,31-33}$. Essas mesmas diretrizes recomendam que todo paciente com DRC avançada deve ser referenciado para um nefrologista.

Entendendo a complexidade do cuidado dos pacientes com fatores de risco ou DRC já instalada e a relevância e necessidade de uma abordagem interdisciplinar, este trabalho teve como objetivo traçar o panorama do acesso ao cuidado, aqui entendido como uma ação integral, dentro da linha de cuidado da DRC, a partir da conduta dos médicos da APS. Sem a pretensão de avaliação e críticas, a partir desse panorama, podem-se discutir propostas para um melhor acompanhamento desse paciente nos níveis primários e especializado de saúde.

\section{Metodologia}

Trata-se de um estudo transversal, realizado com 62 médicos de família da APS do SUS na cidade de Fortaleza, Ceará, no período de agosto de 2011 a fevereiro de 2012.
Foram selecionados aqueles que trabalhavam, no mínimo, há seis meses como médicos de família dos Centros de Saúde da Família do município de Fortaleza; e excluíram-se os com menos de um ano de formado em medicina ou especialização, ou equivalente, em nefrologia. Identificou-se 255 médicos de família atuantes na APS através do cadastro da Secretaria Municipal de Saúde ${ }^{34}$. Para o estudo, foi considerada uma amostra aleatória de 86 profissionais. Desse total, foram excluídos 24 , sendo 15 por não atenderem aos critérios de inclusão e nove por se recusarem a participar da pesquisa.

Aplicou-se um questionário de investigação aos médicos que aceitaram participar espontaneamente da pesquisa. $\mathrm{O}$ instrumento foi preenchido por estes na presença de um coletor de dados treinado.

O questionário consistia de três partes: (1) identificação do médico da atenção primária; (2) capacidade de detectar a DRC em pacientes portadores de fatores de risco (HAS ou DM); e (3) casos clínicos ilustrativos de situações clínicas (lesão renal com TFG normal, DRC leve/moderada e DRC avançada) para avaliar as situações que seriam encaminhadas para a atenção secundária.

A primeira parte (identificação) é constituída por perguntas objetivas sobre: sexo, idade, tempo de formado, especializações adquiridas e vínculos de trabalho (exclusivo ou não na rede pública). Na segunda parte, foram realizadas perguntas abertas sobre quais exames eram solicitados anualmente para os pacientes acompanhados por HAS e DM. Na última parte foram elaborados cinco casos clínicos, a saber:

- Caso 1: HAS e microalbuminúria (lesão renal com TFG normal)

- Caso 2: DM e microalbuminúria (lesão renal com TFG normal)

- Caso 3: HAS com redução leve/moderada da TFG

- Caso 4: DM com redução leve/moderada da TFG

- Caso 5: Paciente com redução acentuada da TFG

Nesses casos, perguntou-se, de forma aberta, qual a conduta a ser realizada pelo médico. Apenas no caso 5 foi perguntado explicitamente se haveria encaminhamento a um profissional da atenção secundária (nefrologista). A intenção de se fazer perguntas abertas foi não sugestionar o entrevistado.

Excetuando as variáveis contínuas (idade, tempo de formado), todas as demais foram trabalhadas como categóricas. Nas perguntas aber- 
tas, foi realizada busca pelas seguintes respostas que caracterizassem o diagnóstico da DRC: solicitação de sumário de urina/microalbuminúria, solicitação de creatinina sérica $(\mathrm{Cr})$, determinação da TFG, encaminhamento ao nefrologista ou para outros profissionais. Essas variáveis foram colocadas como dicotômicas para cada situação específica do questionário.

As variáveis contínuas idade e tempo de formado são apresentadas como média \pm DP e para comparações entre grupos foi utilizado o teste $\mathrm{t}$ não pareado. A normalidade da distribuição dessas variáveis foi avaliada pelo teste de Kolmogorov-Smirnov. As outras foram estudadas como categóricas apresentadas em porcentagem e comparadas pelo teste do qui-quadrado. Foi realizado modelo de regressão logística binária para identificar características do entrevistado (sexo, idade, tempo de formado, especialização e vínculo de trabalho) que fossem relacionadas ao encaminhamento dos pacientes nas diversas situações do questionário. Valores com $\mathrm{p}<0.05$ foram considerados estatisticamente significativos. A análise foi realizada utilizando o SPSS 19.0.

As normas para pesquisa envolvendo seres humanos, estabelecidas pela Resolução 196/96 $6^{35}$ do Conselho Nacional de Saúde, foram respeitadas e o estudo foi aprovado pelo Comitê de Ética em Pesquisa da Universidade de Fortaleza.

\section{Resultados}

\section{Caracterização da população estudada}

Foram entrevistados 62 médicos que trabalham na atenção primária (51.6\% do sexo masculino) com idade média de $37.8 \pm 9.5$ anos. $\mathrm{O}$ tempo médio de formado dos entrevistados era de $11.8 \pm 9.1$ anos. A maioria dos sujeitos (54.8\%) apresentava especialização formal em medicina de família. Vínculo exclusivo com o serviço público foi observado em $77.4 \%$ dos entrevistados.

A maior parte dos médicos solicita dosagem de Cr para pacientes acompanhados por DM ou HAS (88.7 e 93.5\%, respectivamente). Entretanto, quando se considera meios de detecção mais precoces da DRC, sumário de urina/microalbuminúria e medida da TFG, o número de médicos que os realizam reduz de forma significante, especialmente quando consideramos a medida da TFG (Gráfico 1).

Alguma forma de detectar proteína na urina (sumário de urina/microalbuminúria) foi solicitada por aproximadamente $70 \%$ dos entrevistados, sendo mais comum a realização do sumário de urina.

Considerada, atualmente, a melhor forma de medir a função renal e classificar a DRC, a medida da TFG foi lembrada por apenas $8.1 \%$ dos

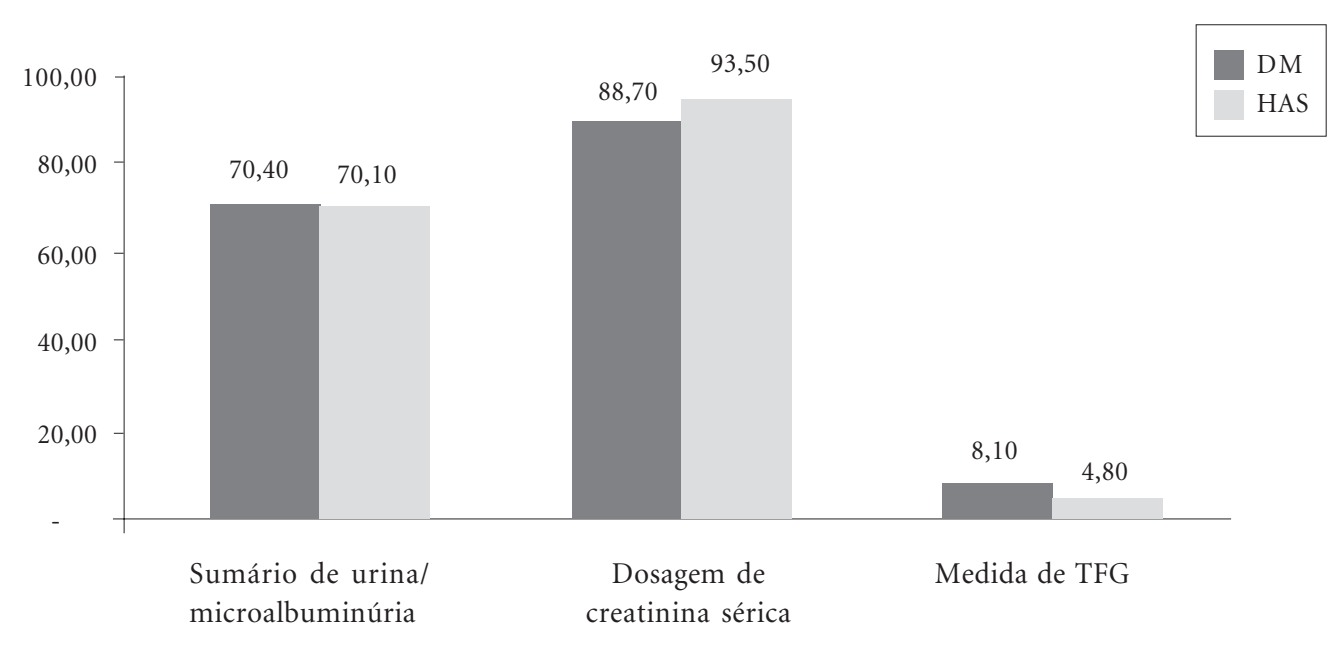

Gráfico 1. Detecção DRC (\%) 
médicos para pacientes com DM e $4.8 \%$ para aqueles pacientes que eram acompanhados por HAS.

Para avaliar o encaminhamento da atenção primária dos pacientes portadores de DRC, foram selecionados casos de pacientes com fatores de risco (DM ou HAS) apresentando (1) lesão renal e TFG normal; (2) redução leve/moderada da TFG; e (3) redução acentuada da TFG.

Na primeira situação, onde o paciente do caso clínico apresentava apenas HAS e lesão renal com TFG normal, 30.6\% dos médicos encaminhariam o paciente à atenção especializada.

Quanto ao caso clínico em que o paciente apresentava HAS e redução leve/moderada da TFG, mais da metade dos médicos (51.2\%) solicitariam que o paciente fosse encaminhado à atenção secundária. Na presente situação, houve uma associação entre a realização da medida da TFG e o encaminhamento à atenção secundária onde $73 \%$ dos que realizavam medida da TFG solicitaram encaminhamento contra $50 \%$ dos que não realizavam medida da TFG $(\mathrm{p}=0.048)$. Apesar de metade dos médicos não encaminharem pacientes com redução leve/moderada da função renal, apenas 19\% solicitariam sumário de urina/microalbuminúria, marcador importante da progressão da DRC.

Mudando o cenário das situações para um paciente diabético ao invés de hipertenso, houve um aumento discreto não significativo do encaminhamento dos pacientes com lesão renal e TFG normal (30.6\% vs. $40.3 \%, \mathrm{p}=0.342)$; enquanto a presença do DM como doença de base não alterou o encaminhamento de pacientes com redução leve/moderada da função renal (51.2\% vs. $53.2 \%, \mathrm{p}=0.857)$.

Apesar da terceira situação estudada representar um caso com redução avançada da função renal, 25.8\% dos médicos da APS não encaminhariam o paciente à atenção especializada. Quanto à abordagem multiprofissional de pacientes hipertensos e diabéticos, apenas 15 (20.5\%) dos médicos de família encaminhavam pacientes diabéticos para outros profissionais não médicos, enquanto 9 (12.3\%) encaminhariam os pacientes hipertensos.

Analisando o perfil dos entrevistados e sua correlação com a detecção da DRC, identificamos que médicos sem vínculo exclusivo com a rede pública mediam mais a TFG, mesmo após ajuste para outras variáveis (OR 1.878 IC 1.031$3.574, \mathrm{p}=0.032$ ). Idade do entrevistado e tempo de formado também foram inversamente associados à detecção da DRC (OR 0.777 IC 0.633-
$0.955, \mathrm{p}=0.016$ e OR 0.732 IC 0.435-0.973, $\mathrm{p}=$ 0.027 , respectivamente) (Tabela 1 ).

Quanto ao encaminhamento à atenção especializada, menor idade e menor tempo de formado dos entrevistados também foram associados com o referenciamento ao nefrologista de pacientes com TFG normal ou redução leve/moderada da TFG (Tabela 2). Não foram encontradas diferenças no perfil do entrevistado quanto ao encaminhamento de pacientes com redução avançada da TFG.

\section{Discussão}

No presente estudo, detectou-se que os médicos da atenção primária, apesar de determinarem pouco a TFG (exame mais sensível e preconizado para a detecção da DRC), sentem necessidade de um encaminhamento precoce desse grupo de usuários. Mais ainda, até um quarto dos médicos entrevistados não encaminhariam pacientes com DRC avançada à atenção secundária. Essas detecções levam a pensar em um modelo de organização dos serviços de saúde onde exista uma aproximação dos níveis primário e especializado, tornando possível o apoio à APS no cuidado aos pacientes com disfunção leve/moderada e no encaminhamento adequado dos casos com disfunção avançada.

$\mathrm{Na}$ detecção da DRC em pacientes de risco, verificou-se baixo nível de solicitação dos exames

Tabela 1. Análise multivariada dos fatores médicos relacionados à detecção dos pacientes com DRC.

\begin{tabular}{lccc}
\hline & $\begin{array}{c}\text { Razão de } \\
\text { chances }\end{array}$ & IC (95\%) & P \\
\hline Idade (anos) & 0.777 & $0.633-0.955$ & 0.016 \\
Tempo de formado (anos) & 0.732 & $0.435-0.973$ & 0.027 \\
Vínculo com rede & 1.878 & $1.031-3.574$ & 0.032 \\
suplementar de saúde & & & \\
\hline
\end{tabular}

Tabela 2. Análise multivariada dos fatores médicos relacionados ao encaminhamento dos pacientes com DR

\begin{tabular}{lccc}
\hline & $\begin{array}{c}\text { Razão de } \\
\text { chances }\end{array}$ & IC (95\%) & P \\
\hline Idade (anos) & 0.682 & $0.578-0.863$ & 0.005 \\
Tempo de formado (anos) & 0.711 & $0.671-0.929$ & 0.024 \\
\hline
\end{tabular}


capazes de detectar precocemente esse distúrbio, especialmente a medida da TFG. A baixa taxa de solicitação desse exame não é explicada pelo custo, pois são exames baratos e amplamente disponíveis nos serviços de atenção primária para o acompanhamento dos portadores de diabetes e hipertensão. Considerando-se a estimativa de que até $1,5 \%$ dos recursos disponíveis ao SUS são gastos, apenas em TRS ${ }^{21}$, com pacientes portadores de DRC avançada e é consenso de que a detecção precoce da DRC pode mudar o curso natural da doença ${ }^{13-16}$, reduzindo morbimortalidade e custos posteriores na atenção de alta complexidade, cabe uma reflexão sobre a reestruturação dos processos de trabalho e a formação de profissionais de saúde no chamado nível primário da atenção $0^{8,12,36,37}$. Desenha-se uma possibilidade da atenção especializada trabalhando próximo ao nível primário, transformando o ato do cuidar.

Nesse sentido, poder-se-ia enfrentar o problema que observamos, onde mais da metade desses profissionais $(51,2 \%)$ referenciam pacientes com DRC em estágio leve ou moderado. Extrapolando dados americanos, teríamos, tomando a capital cearense como exemplo, uma população adulta acima de 20 anos estimada em 180.830 pessoas com DRC nos estágios leve/moderado $(10,8 \%$ da população total nessa faixa etária $)^{38-41}$.

Seguindo a tendência dos dados obtidos, metade dessas pessoas seria encaminhada ao nível especializado. Considerando que os dois níveis de complexidade se apresentam em um desenho ainda verticalizado e fragmentado, o impacto nas agendas do nível especializado pela significativa demanda por encaminhamento no estágio leve/moderado da DRC poderia acarretar em comprometimento da equidade devido ao estrangulamento no acesso dos casos avançados da DRC ao especialista. Esse distanciamento implicaria, também, na possibilidade de retenção destes pacientes pelo nefrologista, sem a garantia da contra referência, rompendo com a lógica do vínculo terapêutico e comprometendo a qualidade do cuidado ${ }^{36}$.

A lacuna entre esses dois níveis da atenção retrata que o arranjo dos serviços de saúde ainda não foi modificado na sua matriz original fragmentada, onde o acesso do usuário é obstaculizado, podendo comprometer avanços no plano da integralidade $e^{37,42}$.

Nesse contexto, a proposta do Modelo de Referência e Apoio Especializado Matricial ${ }^{36}$ seria, à luz do nosso entendimento, o dispositivo que melhor responderia ao problema observado. Esse agenciamento fundamenta o estabelecimento de linhas de cuidado em que projetos terapêuticos, centrados no usuário, são viabilizados numa rede de serviços, otimizando recursos dentro do próprio território da comunidade, em uma permanente discussão conjunta de processos de trabalho e objetivos, pressupondo um conjunto articulado de ações com efetividade comprovada, objetivando uma fundamental e estreita colaboração entre nefrologistas e o nível primário, e entre os profissionais nele inseridos $s^{8,33,36,37}$.

Dessa forma, o cuidado transcenderia um procedimento simplificado e assumiria a forma de uma ação integral, em que as relações não são permeadas pelo domínio, mas pela "con-vivência", facilitando o acesso aos recursos disponíveis ao melhor cuidado possível ${ }^{11,37}$.

Outro achado significativo da pesquisa foi o fato de que 25,8\% dos médicos do nível primário não solicitam uma avaliação do nefrologista nos casos característicos de redução avançada da TFG. Essa situação sinaliza o risco de um incremento na morbimortalidade e nos custos relacionados à visita tardia ao nefrologista, levando-se em conta a alta incidência de comorbidades durante o curso natural da doença na fase avançada, especialmente as relacionadas aos riscos cardiovasculares (DM, HAS e Dislipidemia). Estima-se que entre $30 \%$ a $50 \%$ desses pacientes morrem por doenças cardiovasculares ${ }^{13,19}$. Acresce-se a esse fato, o custo e o sofrimento para familiares e nações, devido ao impacto negativo na qualidade de vida e na autonomia do portador de DRC em fase avançada e a sua (in)capacidade laboral.

Estima-se que a população com DRC avançada que necessita de algum tipo de TRS corresponderia a apenas $0,004 \%$ da população brasileira, com um consumo de, aproximadamente, $1,5 \%$ do orçamento da saúde previsto para o ano de $2012^{21,43}$. Com a condução adequada do portador de DM e HAS ou com DRC diagnosticada desde o início e uma colaboração adequada entre os diversos níveis de complexidade da rede, podese retardar o início da necessidade dialítica, repercutindo em uma redução significativa dos custos médicos ${ }^{13-19}$.

$\mathrm{Na}$ busca de alternativas para essa situação, considerando a disponibilidade e o baixo custo dos métodos diagnósticos precoces e a importância do encaminhamento no tempo adequado ao especialista, a implementação de um dispositivo na lógica do Modelo de Referência e Apoio Matricial proposto por Campos ${ }^{35}$, com experiências exitosas no campo da saúde mental ${ }^{44,45}$, seria capaz de estabelecer um novo tipo de inter-relação 
entre esses dois níveis da atenção com impactos na resolubilidade, considerando seu potencial de articulação em rede e criação de vínculo ${ }^{37}$.

Reforçando essa linha de pensamento, Diamatidis et al. ${ }^{46}$ advogam que modelos de cuidado colaborativos, que incluem os médicos do nível primário e especificam seus papéis e dos nefrologistas no gerenciamento das necessidades dos pacientes com DRC, podem melhorar a qualidade do cuidado e obter melhores resultados.

O presente estudo constatou que médicos mais jovens e com menor tempo de formado solicitam mais exames, como a TFG, e encaminham mais os usuários em estágios leves/moderados da doença. Por um lado, esse fato reflete positivamente em uma maior atenção médica para a DRC como um problema controlável em nível primário. Entretanto, se não for trabalhado o acompanhamento desses usuários, dentro de um sistema não verticalizado, haverá um excesso de demanda com pouca disponibilidade de especialistas e o alto custo da atenção especializada.

Outro achado relevante, considerando a complexidade do cuidado da DRC e o reconhecido papel de uma abordagem interdisciplinar, foi o número muito baixo de encaminhamentos a outros profissionais. Essa situação reflete a negação da possibilidade de construção de novas formas de compreensão e atuação desse problema de saúde, deixando de agir nos paradoxos que o campo da disputa do mundo do trabalho em saúde apresenta ${ }^{12,47,48}$. A literatura ${ }^{12,47,48}$ atenta para a necessidade da reorientação das instituições formadoras de profissionais com atitudes mais ampliadas, capazes de apreender seus pacientes e suas necessidades de modo integral.

Resgata-se aqui a reflexão sobre as propostas de reforma da matriz curricular, da aprendizagem orientada em espaços múltiplos, possibilitando o cruzamento dos diversos saberes, a ética e a responsabilização, na adoção de uma postura de não neutralidade, favorecendo a produção de práticas de cuidado que também valorizem a autonomia do usuário e a universalidade do acesso. A intervenção pedagógica deve confi- gurar-se em um dispositivo que permita mudança na prática profissional para, assim, transpor a relação assistencial direta ${ }^{12,48-50}$.

O estudo apresenta como limitação o número de médicos de família que participaram da pesquisa. Porém, esse quantitativo corresponde a $24,3 \%$ do quadro total de médicos que atuam na ESF no município de Fortaleza. Essa pesquisa também não aprofundou nos fatores que pudessem determinar as condutas aqui observadas, o que demanda o desenvolvimento de outros trabalhos para contemplar essa lacuna. Entretanto, o presente estudo se mostra relevante devido ao restrito número de pesquisas com esse enfoque na literatura.

Em concluso, os dados permitiram observar que os médicos da atenção primária apesar de determinarem pouco a TFG, sentem necessidade de um encaminhamento precoce desse mesmo grupo de usuários. Contudo, até um quarto dos médicos entrevistados não encaminhariam pacientes com DRC avançada à atenção especializada. Também foi possível constatar o baixo número de encaminhamentos de pacientes com DM e HAS a outros profissionais de saúde.

De posse dos achados e entendendo que o nível primário da atenção não é capaz, isoladamente, de dar todas as respostas às demandas da população portadora de fatores de risco ou de DRC já estabelecida, recomenda-se a implantação do dispositivo de "apoio especializado matricial", com a inclusão do especialista em nefrologia, numa lógica do cuidado paciente-centrado; e a necessidade de reorientação das instituições formadoras dos profissionais de saúde para uma atitude menos reducionista. Essas propostas poderiam contribuir para a facilitação do acesso aos diversos recursos e níveis do cuidado, garantindo a integralidade da atenção e a legitimação da APS resolutiva, respondendo à urgência da implementação de um novo arranjo organizacional na produção de novos profissionais e no processo de trabalho, direcionado para a produção de saúde dentro de uma rede dinâmica de serviços e ações. 


\section{Colaboradores}

PFA Pena e AB Libório conceberam o artigo, realizaram levantamento bibliográfico, coleta e análise preliminar de dados e redação e formatação do artigo. AG Silva Júnior orientou as etapas do trabalho, colaborou na revisão da literatura e realizou a revisão final do artigo. PTR Oliveira colaborou na revisão final do artigo. GAR Moreira realizou a revisão, redação e formatação final do artigo.

\section{Referências}

1. Merhy EE. A perda da dimensão cuidadora na produção de saúde: uma discussão do modelo assistencial e da intervenção no seu modo de trabalhar a assistência. In: Campos CR, organizador. Sistema Único de Saúde em Belo Horizonte: reescrevendo o público. São Paulo: Editora Xamã; 1998. p. 103-120.

2. Franco TB, Magalhães Júnior HM. Integralidade na assistência à saúde: a organização das linhas do cuidado. In: Merhy EM, Magalhães Júnior HM, Rimoli J, Franco TB, Bueno WS, organizadores. $O$ trabalho em saúde: olhando e experienciando o SUS no cotidiano. São Paulo: Hucitec; 2003. p. 125-133.

3. Pinheiro R, Mattos RA, organizadores. Gestão em redes: práticas de avaliação, formação e participação na saúde. Rio de Janeiro: Cepesc, UERJ, IMS, Abrasco; 2006.

4. Silva Júnior AG, Mascarenhas MTM. Avaliação da Atenção em Saúde sob a Ótica da Integralidade: Aspectos Conceituais e Metodológicos. In: Pinheiro R, Mattos RA, organizadores. Cuidado: as fronteiras da Integralidade. Rio de Janeiro: Cepesc, UERJ, IMS, Abrasco; 2006a. p. 241-257.

5. Oliveira PTR, Ferla AA, Pinto LF, Batista LV, Pena PFA, Silveira RP, Vieira JR, Negreiros MAMP. Políticas de cuidado da saúde suplementar na região Norte do Brasil: Evidências a partir da análise do cenário regional [projeto de pesquisa]. In: Pereira RC, Silvestre RM, organizadores. Regulação e modelos assistenciais em saúde suplementar: produção científica da Rede de Centros Colaboradores da ANS-2006/2008. Brasília: Organização Pan-Americana da Saúde-Brasil; 2007.

6. Oliveira PTR. Desigualdade Regional e o Território da Saúde na Amazônia. Belém: EDUFPA; 2008.

7. Starfield B. Atenção primária: equilíbrio entre necessidades de saúde, serviços e tecnologia. Brasília: UNESCO, Ministério da Saúde; 2002.

8. Silva Jr AG, Carvalho LC, Silva VMN, Alves MGM, Mascarenhas MTM. Avaliação de redes de atenção à saúde: contribuições da integralidade. In: Pinheiro R, Mattos RA, organizadores. Gestão em Redes: práticas de avaliação, formação e participação na saúde. Rio de Janeiro: Cepesc, UERJ, IMS, Abrasco; 2006.

9. Giovanella L, Mendonça MH. Atenção primária à saúde. In: Giovanella L, Escorel S, Lobato LVC, Noronha JC, Carvalho AI, organizadores. Politicas e Sistema de Saúde no Brasil. Rio de Janeiro: Editora Fiocruz; 2008. p. 575-625.

10. Mendes EV. As redes de atenção à saúde. 2a Edição. Brasília: Organização Pan-Americana de Saúde; 2011.

11. Malta DC, Cecílio LCO, Merhy EE, Franco TB, Jorge AO, Costa MA. Perspectivas da regulação na saúde suplementar diante dos modelos assistenciais. Cien Saude Colet 2004; 9(2):433-444.

12. Pinheiro R, Mattos RA, organizadores. Os sentidos da integralidade na atenção e no cuidado à saúde. $1^{\mathrm{a}}$ Edição. Rio de Janeiro: Cepesc, UERJ, IMS, Abrasco; 2001. 
13. Cleveland DR, Jindal KK, Hirsch DJ, Kiberd BA. Quality of prereferral care in patients with Chronic Renal Insufficiency. Am J Kidney Dis 2002; 40(1):3036.

14. Martínez-Ramírez HR, Martínez BJ, Sanabria LC, Campos ER, Barragán G, Alfaro G, Manzano AMC. Renal function preservation in Type 2 Diabetes Mellitus patients with early nephropathy: A comparative prospective cohort study between Primary Health Care Doctors and a Nephrologist. Am J Kidney Dis. 2006; 47(1):78-87.

15. Francisco ÁLM, De la Cruz JJ, Cases A, Figuera M, Egocheaga I, Górriz JI, Llisterri JI, Marín R, Castelao AM. Prevalencia de insuficiencia renal en Centros de Atención Primaria en España: Estudio EROCAP. Revista Nefrologia 2007; 27(3):300-312.

16. Cueto-Manzano AM, Cortés-Sanabria L, Martinez-Ramirez HR. Management of chronic kidney disease: primary health-care setting, self-care and multidisciplinary approach. Clinical Nephrology 2010; 74(Supl. 1):S99-S104.

17. Lugon JR. Doença Renal Crônica no Brasil: um problema de saúde pública. J Bras Nefrol 2009, 31(Supl. 1):2-5.

18. Bastos MG, Kirsztajn GM. Doença renal crônica: importância do diagnóstico precoce, encaminhamento imediato e abordagem interdisciplinar estruturada para a melhora do desfecho em pacientes ainda não submetidos à diálise. J Bras Nefrol 2011 33(1):93-108

19. Ronksley PE, Hemmelgam BR. Optimizing Care for Patients With CKD. Am J Kidney Dis. 2012; 60(1):133138.

20. Kidney Disease Outcome Quality Initiative-K/DOQI. K/DOQI clinical practice guidelines for chronic kidney disease: evaluation, classification, and stratification. Am J Kidney Dis 2002; 39(Supl. 2):S1-S246.

21. Romão Júnior JE. Doença Renal Crônica: Definição, epidemiologia e classificação. J Bras Nefrol 2004; 36(Supl. 1):1-3.

22. Brasil. Ministério da Saúde (MS). Secretaria de Atenção à Saúde. Prevenção Clínica de Doença Cardiovascular, Cerebrovascular e Renal Crônica. Cadernos de Atenção Básica, 14 (Série A), Normas e Manuais Técnicos. Brasília: MS; 2006.

23. Bastos MG, Bregman R, Kirsztajn GM. Doença Renal Crônica: Frequente e Grave, mas também prevenível e tratável. Rev Assoc Med Bras 2010; 56(2):248 253

24. Brasil. Ministério da Saúde (MS). Secretaria de Vigilância em Saúde. Departamento de Análise de Situação de Saúde. Plano de ações estratégicas para o enfrentamento das doenças crônicas não transmissíveis (DCNT) no Brasil 2011-2022. Brasília: MS; 2011

25. Abegunde DO, Mathers CD, Adam T, Ortegon M, Strong K. The burden and costs of chronic diseases in low-income and middle-income. The Lancet 2007 370(9603):1929-1938.
26. The National Collaborating Centre for Chronic Conditions. Chronic Kidney Disease in Adults: UK Guidelines for Identification, Management and Referral of Adults. [serial on the Internet] [cited 2011 aug 15]. Available from: http://www.rcplondon.ac.uk

27. Tahir MA, Dmitrieva O, Lusignan S, Vlymen J, Chan T, Golmohamad R, Harris K, Tomson C, Thomas $\mathrm{N}$, Gallagher $\mathrm{H}$. Confidence and quality in managing CKD compared with other cardiovascular diseases and diabetes mellitus: a linked study of questionnaire and routine primary care data. BMC Family Practice [serial on the Internet] 2011 Aug [cited 2011 Dec 20]; 12(83): [about 9 p.]. Available from: http://www.biomedcentral.com/1471-2296/12/83

28. Sociedad Española de Nefrologia, Sociedad Española Medicina de Familia e Comunitaria-SEN-SEMFyC. Documento de consenso sobre la enfermedad renal crónica. Barcelona: semfyc ediciones; 2007.

29. Navarro RG. Prevalencia de la Enfermidad Renal Crónica determinada mediante aplicación de ecuaciones predictivas em personas hipertensas atendidas em Atención Primaria. Rev Esp Salud Publica 2009; 83(3):463-469.

30. Sociedade Brasileira de Nefrologia (SBN). [site de Internet] 2012 [acessado 2012 mai 15]. Disponível em: http://www.sbn.org.br

31. Hanset M. L'insuffisance rénale chronique. Le dépistage par le médecin généraliste. Rev Med Brux. 2007; 28(5):465-468.

32. Hernández RS. Enfermedad Renal Crónica: novedades y alcance del seguimiento por el médico de Atención Primaria. Revista Nefrología 2008; 28(Supl. 5):113-117.

33. Lou Arnal LM, Gutiérrez BC, Izquierdo MC, Garcia OG, Alcaine JMT, Garcia SB, Orna JAG, Juliani BB, Hernández-French AS, Grupo de Investigación ERC Aragón. Prevalencia de enfermidad renal crónica en pacientes con diabetes mellitus tipo 2 atendidos en atención primaria. Revista Nefrologia 2010; 30(5):552-556.

34. Secretaria Municipal de Saúde de Fortaleza. [site da Internet] Fortaleza: SMS, 2012 [acessado 2012 abr 12]; Disponível em: http://www.sms.fortaleza. ce.gov.br

35. Brasil. Ministério da Saúde. Conselho Nacional de Saúde. Resolução nº 196 de 10 de outubro de 1996. Diretrizes e Normas Regulamentadoras de Pesquisas Envolvendo Seres Humanos. Diário Oficial da União 1996; 16 out.

36. Campos GWS. Equipes de referência e apoio especializado matricial: um ensaio sobre a reorganizaçäo do trabalho em saúde. Cien Saude Colet 1999; 4(2):393-403

37. Silva Jr AG, Merhy EE, Carvalho LC. Refletindo sobre o Ato de Cuidar da Saúde. In: Pinheiro R, Mattos RA, organizadores. Construção da integralidade: cotidiano, saberes e práticas em saúde. Rio de Janeiro: Cepesc, UERJ, IMS, Abrasco; 2005. p. 113-128. 
38. Saydah S, Eberhardt M, Burrows NR, Williams D, Geiss L, Dorsey R. Prevalence of Chronic Kidney Disease and associated risk factors- United States, 1999-2004. Morbidity and Mortality Weekly Report [serial on the Internet] $2007 \mathrm{Mar}$ [cited $2012 \mathrm{Mar}$ 12]; 56 (8): [about 5 p.]. Available from: www.cdc. gov/nchs/nhanes/Pubs

39. Instituto Brasileiro de Geografia e Estatística. Censo Demográfico 2010. [site na Internet] 2011 [acessado 2011 abr 12]: Disponível em: http://www.ibge.gov.br

40. Cadastro Nacional de Estabelecimentos de Saúde (CNES). [site na Internet]. 2012 [acessado $2011 \mathrm{abr}$ 12]. Disponível em: http://www.cnes.datasus.gov.br

41. Conselho Federal de Medicina. [site na Internet]. 2011 nov [acessado 2012 jan 15]. Disponível em: http://portal.cfm.org.br/imagens/stories/pdf/ demografiamedicanobrasil.pdf

42. Conill EM. Ensaio histórico-conceitual sobre a Atenção Primária à Saúde: desafios para a organização de serviços básicos e da Estratégia Saúde da Família em centros urbanos no Brasil. Cad Saude Publica 2008; 24(Supl. 1):S7-S27.

43. Sesso RC, Lopes AA, Thomé FS, Lugon JR, Santos DR. Relatório do censo brasileiro de diálise de 2010. J. Bras. Nefrol. 2011; 33(4):442-447.

44. Campos GWS, Domitti AC. Apoio matricial e equipe de referência: uma metodologia para gestão do trabalho interdisciplinar em saúde. Cad Saude Publica 2007; 23(2):399-407.

45. Dimenstein $M$, Severo AK, Brito M, Pimenta AL, Medeiros V, Bezerra E. O Apoio Matricial em Unidades de Saúde da Família: experimentando inovações em saúde mental. Saúde Soc. São Paulo 2009; 18(1):63-74.

46. Diamantidis CJ, Powe NR, Jaar BG, Greer RC, Troll MU, Boulware LE. Primary Care-Specialist collaboration in the care of patients with Chronic Kidney Disease. Clin J Soc Nephrol 2011; 6(2):93-108.
47. Merhy EE. Gestão da produção do cuidado e clínica do corpo sem órgãos: novos componentes dos processos de produção do cuidado em saúde. UFRJ [artigo na Internet] 2007 [acessado 2012 mar 15]: [cerca de 15 p.]. Disponível em: http://www.hucff.ufrj.br/ micropolítica/

48. Ceccim RB, Ferla AA. Residência Integrada em Saúde: uma resposta da formação e desenvolvimento profissional para a montagem do projeto de integralidade da atenção à saúde. In: Pinheiro R, Mattos RA, organizadores. Construção da integralidade: cotidiano, saberes e práticas em saúde. Rio de Janeiro: Cepesc, UERJ, IMS, Abrasco; 2005. p. 211-226.

49. Rimoli J. Análise de uma intervenção pedagógica em centros de saúde e seu impacto na assistência e na gestão. In: Merhy EM, Magalhães Júnior HM, Rimoli J, Franco TB, Bueno WS, organizadores. O trabalho em saúde: olhando e experienciando o SUS no cotidiano. São Paulo: Hucitec; 2003. p. 199-296.

50. Oliveira GS, Koifman L. A busca da integralidade nas práticas de saúde e a diversificação dos cenários de aprendizagem: o direcionamento do curso de Medicina da UFF. In: Pinheiro R, Mattos RA, organizadores. Cuidado: as fronteiras da Integralidade. Rio de Janeiro: Cepesc/UERJ, Abrasco; 2006. p. 307-319.

Artigo apresentado em 30/04/2012

Aprovado em 17/07/1012

Versão final apresentada em 30/08/2012 